Zbl Arbeitsmed 2015 $\cdot 65: 299-300$

DOI 10.1007/s40664-015-0058-4

Online publiziert: 20. Oktober 2015

๑) Springer-Verlag Berlin Heidelberg 2015

CrossMark

\section{J. Schneider}

Institut und Poliklinik für Arbeits- und Sozialmedizin, Universitätsklinikum

Gießen und Marburg, Standort Gießen, Gießen, Deutschland

\title{
Laudatio auf Professor (em.) Dr. med. Hans-Joachim Woitowitz
}

\section{Jahre}

Am 18.10.2015 feiert Herr Professor Dr. med. Hans-Joachim Woitowitz, langjähriger Direktor des Instituts und der Poliklinik für Arbeits- und Sozialmedizin der Justus-Liebig-Universität Gießen, seinen 80. Geburtstag. Zu diesem Anlass sei ihm dieses Heft gewidmet. Herr Professor Woitowitz wurde 1935 in Allenstein/Ostpreußen geboren. Nach dem Abitur 1955 begann er mit dem Studium der Medizin zunächst an der Universität in Marburg und wechselte anschließend nach Köln, wo er nach seiner Medizinal-Assistentenzeit 1963 zum Arzt approbiert wurde. Zwischenzeitlich promovierte er $1961 \mathrm{mit}$ der Arbeit: „Über optische Phänomene bei der zyklothymen Depression“.

\section{Wissenschaftliche Tätigkeit}

Erste Station seiner wissenschaftlichen Tätigkeit war seine Assistentenzeit von 1963 bis 1965 an der namhaften medizinischen Klinik der Universität zu Köln unter den Ordinarien der Herren Professoren Dr. med. Dr. h.c. mult. H.W. Knipping und Prof. Dr. Groß. Anfang Oktober 1965 folgte er Herrn Prof. Dr. H. Valentin, dem damaligen Direktor des Instituts für Arbeits- und Sozialmedizin und der Poliklinik für Berufskrankheiten an die Universität Erlangen-Nürnberg. Dort war Herr Professor Woitowitz bis 1972 als wissenschaftlicher Assistent und bis zu seinem Ausscheiden 1974 als Oberassistent tätig. Während dieser Zeit erhielt er 1969 die Anerkennung als Facharzt für Innere Medizin mit der Zusatzbezeichnung „Arbeitsmedizin“ sowie 1971 die Venia Legendi der Medizinischen Fakultät als Privatdozent für die beiden Fächer Arbeits- und Sozialmedizin. Am 14.10.1974 erfolgte die Berufung zum Professor des Instituts und der Poliklinik für Arbeitsmedizin - seit 1976 Arbeits- und Sozialmedizin - des Medizinischen Zentrums für Ökologie des Klinikums an der Justus-LiebigUniversität in Gießen.

Bereits während seiner Erlangener Zeit hat sich Herr Professor Woitowitz wissenschaftlich speziell mit den krebserzeugenden Risiken nach beruflicher Asbesteinwirkung auseinandergesetzt, der Thematik seiner Habilitationsschrift, die ihm, nachdem er bereits erstmals 1969 den E.W. Baader-Preis für seine Arbeit über die Blutgasanalyse während körperlicher Belastung verliehen bekam, die gleiche Ehrung 1971 erneut zuteilwerden ließ. Dieses Forschungsgebiet hat er trotz einigen starken Gegenwindes gradlinig und erfolgreich weiterverfolgt.

Wissenschaftlich geprägt ist die Zeit an der Gießener Justus-Liebig-Universität ferner durch seine Aktivitäten bei der Risikobeurteilung allgemeiner krebserzeugender Prinzipien und deren Einstufung in der Arbeitswelt und Umwelt. Stellvertretend zu nennen sind neben Asbest die polyzyklischen aromatischen Kohlenwasserstoffe oder die Analyse von Biomarkern nach beruflicher Gefährdung bei vormals im Uranbergbau tätigen Arbeitnehmern. Weitere wissenschaftliche Schwerpunkte beinhalten die Kausalanalyse industrieller Latenzschäden, obstruktive Atemwegserkrankung durch allergisierende oder chemisch irritative Gefahrstoffe, die sozial- und arbeitsmedizinische Epidemiologie und Begutachterkunde, um nur einige zu nennen.

\section{Aufgaben und Ämter}

So ist es naheliegend, dass Herrn Professor Woitowitz infolge seines profunden Wissens Aufgaben und Ämter angetragen wurden, denen er sich mit großem Engagement widmete. So leitete er seit 1983 in der Senatskommission zur Prüfung gesundheitsschädlicher Arbeitsstoffe der DFG die Arbeitsgruppe „Festlegung von Grenzwerten für Stäube“, seit 1989 war er stellvertretender Vorsitzender der Arbeitsgruppe „Krebsbekämpfung am Arbeitsplatz" des Gesamtprogramms zur Krebsbekämpfung beim Bundesministerium für Gesundheit. Im Jahr 1991 wurde er zum Vorsitzenden der Sektion „Berufskrankheiten “ des Ärztlichen Sachverständigen-Beirates beim Bundesministerium für Arbeit- und Sozialordnung berufen. In dieser Zeit sind neue Berufskrankheiten aufgenommen worden, die eine Fortentwicklung des BK-Rechtes bedeuten. Stellvertretend hierfür seien die Berufskrankheiten mit Dosimetrisch-probabilistischen Lösungsansätzen und die Synkanzerogenese genannt. Den Vorsitz führte Herr Professor Woitowitz ebenfalls beim interministeriellen Beirat der zentralen Betreuungsstelle Wismut, Hauptverband der gewerblichen Berufsgenossenschaften sowie im Sachverständigenkreis Prävention im Arbeits- und betrieblichen Gesundheitsschutz beim Bundesministerium für Bildung, Wissenschaft, Forschung und Technologie. Als Zeichen besonderer Ehre und Fachkompe-

Dieser Beitrag gehört zum Jubiläumsheft und ist Herrn Prof. Woitowitz zum 80. Geburtstag gewidmet. 
tenz zu werten ist der an ihn 1979 ergangene Ruf auf das Ordinariat des Instituts und der Poliklinik für Arbeits-, Sozialmedizin und Sozialhygiene der Universität Köln, den Herr Professor Woitowitz jedoch ablehnte. Seine Bemühungen um die Motivation, Fort- und Weiterbildung junger Kolleginnen und Kollegen auf arbeitsund sozialmedizinischem Gebiet führten 1978 zur Gründung der Hessischen, später Hessisch-Thüringischen Akademie für Arbeits-, Sozial- und Umweltmedizin, deren Präsident Herr Professor Woitowitz langjährig war. Mit Überleitung der Akademie an die Landesärztekammer in Hessen ist Herr Professor Woitowitz langjähriger Sektionsvorsitzender.

Herr Professor Woitowitz war ein halbes Jahrhundert Wegbereiter der hessischen und bundesdeutschen Arbeitsmedizin. So hat er wesentliche Impulse zur Weiterentwicklung und Gestaltung der Arbeitsmedizin, -sicherheit und zum Sozialrecht gegeben. Als Mitglied der ständigen Konferenz „Arbeitsmedizin“ der Bundesärztekammer, des Beirates des Präsidiums sowie als Gutachter und Prüfer in den Weiterbildungsgremien der Landesärztekammer Hessen für die Arbeitsund Sozialmedizin war er aufopfernd tätig und hat initiative Wege beschritten, die zur Verbesserung der Weiterbildung führten. Etwa 50 Kolleginnen bzw. Kollegen haben die arbeitsmedizinische und zum Teil auch die sozialmedizinische Weiterbildung absolviert und damit die Anerkennung als Fachärztin/Facharzt für Arbeitsmedizin bzw. die Zusatzbezeichnung Sozialmedizin erworben.

\section{Verdienste und Ehrungen}

Im Jahr 1992 wurden die Verdienste, die wesentlich zum hohen beruflichen Standard der Arbeitsmedizin beigetragen haben, durch die Verleihung der Ernst von Bergmann-Plakette der Bundesärztekammer gewürdigt. Internationale Anerkennung seiner Leistung erfuhr Herr Professor Woitowitz 1987 durch die Berufung als „Fellow of the Collegium Ramazzini“, Bologna sowie 1979 als „Member of the Board of the Ramazzini Institute for Occupational and Environmental Health Research", Salomons/Washington.
Für seine Verdienste wurde Herr Professor Woitowitz mit dem Bundesverdienstkreuz am Bande, der Ehrenplakette der Landesärztekammer Hessen in Silber und dem international anerkannten Ramazzini Award geehrt. Die höchste Auszeichnung der deutschen Ärzteschaft erhielt er mit der Paracelsus-Medaille im Jahr 2013. Das Postulat von Paracelsus „Die Dosis macht das Gift" war für Herrn Professor Woitowitz zentrale Grundlage für die auch in das Berufskrankheitenrecht aufgenommenen Dosis-WirkungsBeziehungen.

\section{Publikationen}

Nicht unerwähnt bleiben dürfen die im internationalen und nationalen Schrifttum publizierten Ergebnisse seiner Forschungsaktivitäten. So ist er Autor bzw. Koautor von weit über 500 wissenschaftlichen Veröffentlichungen.

\section{Engagement nach Ende der aktiven Dienstzeit}

Die aktive Dienstzeit von Herrn Professor Dr. med. Hans-Joachim Woitowitz endete am 31.03.2004. Zeit für Entspannung bleibt ihm trotzdem nicht. Aufgrund seiner hohen Fachkompetenz ist sein Rat in Gremien, Ausschüssen und in Ministerien sehr gefragt. Herr Professor Woitowitz veranstaltete bis vor Kurzem ärztliche Fortbildungsveranstaltungen und ist ein viel gefragter Referent. In Büchern und in anderen Medien weist er auf die langen und oft schrecklichen Leidenswege der an Berufskrebs Erkrankten und deren Angehörige hin. Sein ärztliches Ethos veranlasst ihn, im Rahmen der Sozialgerichtsbarkeit schwierigste Kausalzusammenhangsfragestellungen aufzuklären sowie sein reichhaltiges Erfahrungswissen auch bei der Beratung von Selbsthilfegruppen einzubringen. Erst im 80. Lebensjahr reduziert Herr Professor Woitowitz langsam seine Aktivitäten, um mehr Zeit für seine Familie zu gewinnen. Herr Professor Woitowitz hat langjährig ein national und international renommiertes Institut und eine Poliklinik geleitet, an deren Weiterbestand ihm sehr gelegen ist.

\section{Gratulation und Würdigung}

Die ehemaligen und derzeitigen Mitarbeiterinnen und Mitarbeiter des Instituts und der Poliklinik für Arbeits- und Sozialmedizin möchten anlässlich der Vollendung des 80. Geburtstages die herzlichsten Glückwünsche aussprechen.

Mit unserer Gratulation, die Glückwünsche gelten auch für Deine Gemahlin Rotraud, die die Gestaltung Deines Lebensweges maßgeblich mitgetragen hat und als wissenschaftliche Mitarbeiterin auch langjährig in der Abteilung tätig war, möchten wir Dir für die weitere Zukunft alles Gute und insbesondere Gesundheit wünschen. Ad multos annos.

Achim Schneider

\section{Korrespondenzadresse}

Prof. Dr. J. Schneider

Institut und Poliklinik für Arbeits- und

Sozialmedizin

Universitätsklinikum Gießen und Marburg,

Standort Gießen

Aulweg 129/III, 35385 Gießen

joachim.schneider@arbmed.med.uni-giessen.de 